\title{
[2] Tracer Diffusion by Six-Jump-Cycles in Nonstoichiometric B2 Intermetallic Compounds
}

\author{
K.L.Gosain ${ }^{1}$, D.K.Chaturvedi ${ }^{1}$, I.V.Belova ${ }^{2}$ and G.E.Murch ${ }^{2}$ \\ ${ }^{1}$ Department of Physics, Kurukshetra University \\ Kurukshetra-136119, \\ India \\ ${ }^{2}$ Diffusion in Solids Group, School of Engineering \\ The University of Newcastle, Callaghan, NSW 2308, \\ Australia
}

Keywords: Correlation Factors, Intermetallics, Ordered Alloys, Tracer Diffusion

\begin{abstract}
Tracer diffusion in non-stoichiometric B2 intermetallic compounds having antistructural disorder is investigated using the six-jump-cycle (6JC) as a fundamental diffusion unit. For non-stoichiometric compositions, the antistructural atoms are assumed to be isolated and located at one of the six [110]-type and [100]-type sites (as only these sites are involved in the $6 \mathrm{JC}$ or $2 \mathrm{JC}$ ). The jump frequencies for the $6 \mathrm{JC}$ involving a perfectly ordered configuration are calculated in terms of a four-frequency-model, using the meanfirst-passage concept of Arita et al. The jump frequency of an antistructural atom at [110] or [100]-type sites is taken to be the harmonic mean of frequencies of two successive nearestneighbour jumps of the same kind of atoms. The expressions for the tracer diffusion coefficients are derived for both atomic components at deviations from stoichiometry, assuming that the $6 \mathrm{JC}$ mechanism is valid. The results are compared with Monte Carlo simulations based on single vacancy jumps and found to be in fair agreement for compositions close to stoichiometry.
\end{abstract}

\section{Introduction}

Since some intermetallic compounds offer properties of technological interest such as low density, a high strength at elevated temperatures and a high corrosion resistance [1], there has been considerable and sustained interest in the study of diffusion kinetics in these compounds. Many intermetallic compounds that are attractive for technological applications take the $\mathrm{B} 2(\mathrm{CsCl})$ structure. Accordingly, much effort has been devoted to exploring diffusion kinetics in this particular structure.

The basic diffusion mechanism in pure metals is very well established and for the most part consists of vacancy-mediated nearest neighbour jumps. The detailed mechanism of diffusion in B2 intermetallic compounds is much more complex because of the ordered structure itself and because of the additional energy involved in any mechanism that would destroy order, even if only temporarily. For B2 intermetallic compounds showing only antistructural disorder the nearest neighbour vacancy mechanism still occurs, but at high levels of order the vacancy is confined to such a limited number of paths that new concatenated mechanisms can then be identified. The most energetically favourable of these is the six-jump cycle (6JC) [2], a cycle in which the vacancy migrates along a specific path of six nearest-neighbour jumps. After the six effective jumps of the vacancy, the order in the crystal is restored but there has been net transport of matter. Several reviews of the diffusion mechanisms and associated theory in B2 intermetallics have been provided recently $[3,4]$. 
Computer simulations of various models that start only with the basic vacancy mechanism clearly show the emergence of the $6 \mathrm{JC}$ as the level of order increases [5,6]. Historically, theoretical formalisms based on single vacancy jumps have had difficulty in describing the tracer correlation factors at high levels of order, see the review [4]. A different starting point for a tracer diffusion formalism is to commence with the $6 \mathrm{JC}$ itself as the basic unit of the diffusion process. The original or 'pure' $6 \mathrm{JC}$, by itself, does not allow for the participation of existing antistructural atoms produced either by non-stoichiometry (extrinsic defects) or by thermal activation. A logical extension is the antistructural atom-assisted 6JC mechanism (or A6JC). This mechanism consists of a 'pure' $6 \mathrm{JC}$ where the presence of an antistructural atom in a next nearest neighbour site of the vacancy lowers the activation energy barrier. This antistructural atom is not involved in any exchange with the vacancy within the 6JC. Domian and Aaronson [7] were the first to suggest such an approach to tracer diffusion in B2 intermetallics but their formalism concentrated only on the jump frequency and not tracer correlation effects.

Belova and Murch [8-10] extended this concept to describe tracer and collective diffusion in non-stoichiometric B2 intermetallic compounds by considering the antistructural atom as an 'impurity' and using the $6 \mathrm{JC}$ as the diffusion unit in an analogue of the well known five-frequency model for impurity diffusion via single vacancy jumps. They derived expressions for tracer and collective correlation factors and tracer and chemical diffusivities and obtained excellent agreement with results obtained Monte Carlo computer simulation of the Ising alloy model.

In the present paper, we have calculated tracer correlation factors in a non-stoichiometric and partially ordered B2 intermetallic binary compound. The linear response theory described by Allnatt and Lidiard [11] has been used to express phenomenological transport coefficients for the tracer on the two sub-lattices. The required conditional probability functions are evaluated through kinetic equations where the $6 \mathrm{JC}$ mechanism is used as a fundamental unit for diffusion. The results of tracer correlation factors for both atomic components at deviations from stoichiometry are compared with Monte Carlo simulation obtained for the four-frequency model. The results are also compared with those obtained from a previous calculation [12], where the coupling between the two sub-lattices was considered through random nearest-neighbour vacancy jumps only.

\section{Average jump frequency for an isolated 6JC}

In the Ising alloy model, the occurrence of order is due to the imposed interactions between the atoms $[13,14]$. In the simplified 'four-frequency' model (where there is no explicit interaction between atoms except one atom per site), the two sub-lattices exist a priori [15]. Each sub-lattice is considered as a random alloy but in general the two sub-lattices take different compositions. We consider sublattice- 1 as the home sub-lattice of atoms of type B and sublattice-2 as the home sublattice of atoms of type $A$. Then $B_{1}$ and $A_{1}$ respectively denote the regular and antistructural atoms on sublattice-1 whereas $A_{2}$ and $B_{2}$ respectively denote the regular and antistructural atoms on sublattice-2. With two types of vacancies $\left(V_{1}=\right.$ vacancy on sublattice- 1 only and $V_{2}=$ vacancy on sublattice-2 only), there will be four jump frequencies denoted by $\mathrm{W}_{\mathrm{B} 1} \equiv \mathrm{W}_{\mathrm{B}}^{1 \rightarrow 2}$, the atom-vacancy exchange frequency when a B atom is on sublattice-1 and a vacancy is at its nearest-neighbour site (on sublattice-2). Likewise, we define $\mathrm{W}_{\mathrm{A} 1} \equiv \mathrm{W}_{\mathrm{A}}^{1 \rightarrow 2}, \mathrm{~W}_{\mathrm{A} 2} \equiv \mathrm{W}_{\mathrm{A}}^{2 \rightarrow 1}, \mathrm{~W}_{\mathrm{B} 2} \equiv \mathrm{W}_{\mathrm{B}}^{2 \rightarrow 1}$. Similarly for the tracer atom, we have $\mathrm{W}_{\mathrm{T} 1} \equiv \mathrm{W}_{\mathrm{T}}^{1 \rightarrow 2}$ and $\mathrm{W}_{\mathrm{T} 2} \equiv \mathrm{W}_{\mathrm{T}}^{2 \rightarrow 1}$ depending upon whether the tracer is on sublattice-1 or sublattice-2 respectively. For the very dilute vacancy and tracer components, the species occupations for the four-component system can be easily obtained [15].

In the 6JC mechanism, starting from a fully ordered configuration, the vacancy progressively disorders the structure in its first three effective jumps and in its next three effective jumps the vacancy progressively re-orders the lattice resulting in the fully ordered configuration once again. By the end of the sequence of six (effective) vacancy jumps, diffusion has occurred on the same sub-lattice. For a perfectly ordered configuration, the 6JC frequency of regular atom (an 
A-atom on the sublattice- 2 or a B-atom on the sublattice-1) is calculated in terms of the fourfrequency model, using the mean first passage concept of Arita et al. [16] by finding the expectation time for a vacancy to complete the cycle. The inverse of this expectation time is the average jump frequency for the $6 \mathrm{JC}$. For an isolated $6 \mathrm{JC}$, the jump frequency for the structured atom is $(1 / 48)$ of this average frequency [8-10]. Following Arita et al. [16], we thus obtain:

$$
v_{\mathrm{A} 2} \equiv v_{\mathrm{B} 1}=\frac{\alpha^{3}\left(4 \alpha^{2}+6 \alpha+3\right)}{\left(4 \alpha^{7}+48 \alpha^{6}+364 \alpha^{5}+1360 \alpha^{4}+1325 \alpha^{3}+2232 \alpha^{2}+1152 \alpha+288\right)}
$$

Here $\alpha$ is the ratio of the jump frequencies of the regular and antistructural atoms of the same type. That is:

$$
\alpha=\frac{\mathrm{W}_{\mathrm{B} 1}}{\mathrm{~W}_{\mathrm{B} 2}}=\frac{\mathrm{W}_{\mathrm{A} 2}}{\mathrm{~W}_{\mathrm{A} 1}}
$$

For the evaluation of the jump frequency for the antistructural atom we observe that when an antistructural atom is at [110]-type or [100]-type sites relative to the vacancy, after two successive nearest-neighbour vacancy-atom exchanges, the resulting configuration has the same energy as the initial one and the net displacement of the antistructural atom is non-zero. By its very nature, the frequency of this $2 \mathrm{JC}$ is much greater than that of the $6 \mathrm{JC}$ resulting from the same energy configuration. The jump frequency for [110]-2JC or [100]-2JC involving antistructured atom can be taken to be the harmonic mean of the individual jump frequencies of two successive N-N jumps of that kind of atoms [17]. Thus, we have:

$$
v_{\mathrm{B} 2} \equiv v_{\mathrm{A} 1}=\frac{2 \alpha}{(1+\alpha)}
$$

\section{Phenomenological coefficients for the tracer}

It has been recognized that when matter transport is governed by the $6 \mathrm{JC}$ mechanism alone, diffusion on each sub-lattice is independent of the other. Therefore, we consider only one sublattice, say, sublattice-1 and obtain the results for the other sub-lattice by interchanging the subscript 1 with 2. As explained earlier, the tracer diffuses at the end of the sequence of six (effective) vacancy jumps when it is at [110]-type or at [100]-type sites relative to the vacancy. We denote by $v_{\mathrm{T} 1}^{1}$ the effective tracer frequency corresponding to the single isolated [110]-cycle and by $v_{\mathrm{T} 1}^{0}$ the effective frequency corresponding to single isolated [100]-cycle (straight or bent). There are 12 equivalent [110]-type sites, denoted by the coordination number $Z_{1}$, that give the tracer displacement $S_{1}=\sqrt{2}$ a at the end of the $6 \mathrm{JC}$ involving these sites. Similarly there are 6 equivalent [100]-type sites, denoted by $Z_{0}$, that give tracer displacement $S_{0}=a$ at the end of [100]-straight or bent cycles. Here, a is the cube length.

We use the linear response formalism described by Allnatt and Lidiard [11] to evaluate the phenomenological transport coefficients for the tracers in B2 intermetallic compounds. The phenomenological transport coefficient $\mathrm{L}_{\mathrm{T} 1 \mathrm{~T} 1}$ (for sublattice-1) is divided into an uncorrelated part, $\mathrm{L}_{\mathrm{T} 1 \mathrm{~T} 1}^{(0)}$, and a correlated part, $\mathrm{L}_{\mathrm{T} 1 \mathrm{~T} 1}^{(1)}$. The uncorrelated part can be expressed as:

$$
\begin{aligned}
\mathrm{L}_{\mathrm{T} 1 \mathrm{~T} 1}^{(0)} & =\left(\frac{\mathrm{N}}{6 \mathrm{kT}}\right)\left[\mathrm{Z}_{1} \mathrm{~S}_{1}^{2} v_{\mathrm{T} 1}^{1}+2 \mathrm{Z}_{0} \mathrm{~S}_{0}^{2} v_{\mathrm{T} 1}^{0}\right] \mathrm{C}_{\mathrm{V} 1} \mathrm{C}_{\mathrm{T} 1} \\
& =\left(\frac{2 \mathrm{Na}^{2}}{\mathrm{kT}}\right)\left[2 v_{\mathrm{T} 1}^{1}+v_{\mathrm{T} 1}^{0}\right] \mathrm{C}_{\mathrm{V} 1} \mathrm{C}_{\mathrm{T} 1}
\end{aligned}
$$


The factor 2 is multiplied in the second term to account for straight and bent [100]-cycles. Here $\mathrm{N}$ is the number of lattice sites per unit volume, $\mathrm{k}$ is the Boltzmann constant and $\mathrm{T}$ is the absolute temperature. $\mathrm{C}_{\mathrm{V} 1}$ and $\mathrm{C}_{\mathrm{T} 1}$ respectively denote the vacancy and tracer fractional site-concentrations on the sublattice-1.

We further consider that the tracer makes a correlated random walk on a sub-lattice involving only [100]-type or [110]-type sites relative to the vacancy (since only these sites are involved in the $6 \mathrm{JC}$ mechanism) and also the fact that effective single $6 \mathrm{JC}$ frequencies for the straight and bent [100]- cycles are the same. The expression for $\mathrm{L}_{\mathrm{T} 1 \mathrm{T1}}^{(1)}$ can be written as:

$$
\begin{aligned}
\mathrm{L}_{\mathrm{T} 1 \mathrm{~T} 1}^{(1)}=-\left(\frac{\mathrm{N}}{3 \mathrm{kT}}\right)\left[v_{\mathrm{T} 1}^{1} v_{\mathrm{T} 1}^{1} \sum_{\mathrm{S}_{1}, \mathrm{~S}_{1}^{0}} \mathrm{~S}_{1} \cdot \mathrm{S}_{1}^{0} \phi\left(\mathrm{S}_{1}: \mathrm{S}_{1}^{0}\right)+2 v_{\mathrm{T} 1}^{1} v_{\mathrm{T} 1}^{0} \sum_{\mathrm{S}_{1}, \mathrm{~S}_{0}^{0}} \mathrm{~S}_{1} \cdot \mathrm{S}_{0}^{0} \phi\left(\mathrm{S}_{1}: \mathrm{S}_{0}^{0}\right)\right. \\
\left.+2 v_{\mathrm{T} 1}^{0} v_{\mathrm{T} 1}^{1} \sum_{\mathrm{S}_{0}, \mathrm{~S}_{1}^{0}} \mathrm{~S}_{0}^{0} \cdot \mathrm{S}_{1}^{0} \phi\left(\mathrm{S}_{0}: \mathrm{S}_{1}^{0}\right)+4 v_{\mathrm{T} 1}^{0} v_{\mathrm{T} 1}^{0} \sum_{\mathrm{S}_{0}, \mathrm{~S}_{0}^{0}} \mathrm{~S}_{0}^{0} \cdot \mathrm{S}_{0}^{0} \phi\left(\mathrm{S}_{0}: \mathrm{S}_{0}^{0}\right)\right]
\end{aligned}
$$

where we have defined, for example,

$$
\varphi\left(\mathrm{S}_{1}: \mathrm{S}_{0}^{0}\right)=\int_{0}^{\infty} \mathrm{dt} \sum_{\lambda} \psi_{\mathrm{V} 1 \mathrm{~T} 1: \mathrm{V} 1 \mathrm{~T} 1}\left(\lambda, \lambda-\mathrm{S}_{1} ; \mathrm{t}: \lambda^{0}, \lambda^{0}-\mathrm{S}_{0}^{0} ; \mathrm{t}=0\right)
$$

Here $\psi_{\mathrm{VITl}: \mathrm{V} 1 \mathrm{T1}}\left(\lambda, \lambda-\mathrm{S}_{1} ; \mathrm{t}: \lambda^{0}, \lambda^{0}-\mathrm{S}_{0}^{0} ; \mathrm{t}=0\right)$ is the conditional probability of finding at time $t$ the vacancy at site $\lambda$ and the tracer at its [110]-type site $\left(\lambda-S_{1}\right)$ when initially the vacancy was at any site $\lambda^{0}$ and tracer at [100]-type site $\left(\lambda^{0}-S_{0}^{0}\right)$ on the same sub-lattice. In order to proceed further, we follow Chaturvedi and Allnatt [18,19] and define the Fourier transform as:

$$
\widetilde{\phi}\left(\mathrm{k}: \mathrm{S}^{0}\right)=\sum_{\mathrm{r}} \mathrm{e}^{\mathrm{ik} . \mathrm{r}} \phi\left(\mathrm{r}: \mathrm{S}^{0}\right)
$$

and the inverse Fourier transform as:

$$
\phi\left(r: S^{0}\right)=\left(\frac{a}{2 \pi}\right)^{3} \int d^{3} k e^{-i k . r} \tilde{\phi}\left(k: S^{0}\right)
$$

where the integrations are over the Brillouin zones in cubic lattice. Using these definitions, Eqn. 5 can be expressed as

$$
\mathrm{L}_{\mathrm{T} 1 \mathrm{~T} 1}^{(1)}=-\left(\frac{2 \mathrm{Na}^{2}}{\mathrm{kT}}\right)\left[2 v_{\mathrm{T} 1}^{1} v_{\mathrm{T} 1}^{1} \mathrm{U}_{1}\left(: \mathrm{S}_{1}^{0}\right)+4 v_{\mathrm{T} 1}^{1} v_{\mathrm{T} 1}^{0} \mathrm{U}_{0}\left(: \mathrm{S}_{1}^{0}\right)+v_{\mathrm{T} 1}^{0} v_{\mathrm{T} 1}^{1} \mathrm{U}_{1}\left(: \mathrm{S}_{0}^{0}\right)+2 v_{\mathrm{T} 1}^{0} v_{\mathrm{T} 1}^{0} \mathrm{U}_{0}\left(: \mathrm{S}_{0}^{0}\right)\right]
$$

In the above we have defined, for example:

$$
\mathrm{U}_{0}\left(: \mathrm{S}_{1}^{0}\right)=8 \mathrm{i}\left(\frac{\mathrm{a}}{2 \pi}\right)^{3} \int \mathrm{d}^{3} \mathrm{k} \operatorname{sink}_{\mathrm{x}} \mathrm{a} \tilde{\varphi}\left(\mathrm{k}: \mathrm{S}_{1}^{0}\right)
$$

and

$$
\mathrm{U}_{1}\left(: \mathrm{S}_{1}^{0}\right)=8 \mathrm{i}\left(\frac{\mathrm{a}}{2 \pi}\right)^{3} \int \mathrm{d}^{3} \mathrm{k} \sin _{\mathrm{x}} \mathrm{a} \operatorname{cosk}_{\mathrm{y}} \mathrm{a} \tilde{\varphi}\left(\mathrm{k}: \mathrm{S}_{1}^{0}\right)
$$

Here we have used symmetry and taken a particular value of the initial vectors $\mathrm{S}_{1}^{0}$ and $\mathrm{S}_{0}^{0}$, say $\mathrm{S}_{1}^{0}=\mathrm{a}(\hat{\mathrm{x}}+\hat{\mathrm{y}})$ and $\mathrm{S}_{0}^{0}=\mathrm{ax}$. 


\section{Kinetic Equation}

In the microscopic study of diffusion in solids the kinetic equation approach has proved very useful (see, Allnatt and Lidiard [11]; Chaturvedi and Allnatt [18,19]; Sharma et al. [20,21]). The rate equation for the probability function $\psi_{\mathrm{VIT1}}(\lambda, \lambda-\mathrm{r} ; \mathrm{t}:)$, which is the conditional probability of finding at time $t$ the vacancy at site $\lambda$ and the tracer at site $(\lambda-r)$ when the system was in the given initial state, can be written as:

$$
\begin{aligned}
\frac{\mathrm{d} \psi_{\mathrm{V} 1 T 1}(\lambda, \lambda-\mathrm{r})}{\mathrm{dt}} & =\left\{v_{\mathrm{T} 1}^{1} \theta_{1}(\mathrm{r})+2 v_{\mathrm{T} 1}^{0} \theta_{0}(\mathrm{r})\right\}\left[\psi_{\mathrm{V} 1 \mathrm{~T} 1}(\lambda-\mathrm{r}, \lambda)-\psi_{\mathrm{V} 1 \mathrm{~T} 1}(\lambda, \lambda-\mathrm{r})\right] \\
& +\sum_{\mathrm{n}} \sum_{\mathrm{r}^{\prime} \neq \mathrm{r}}\left\{v_{\mathrm{n} 1}^{1} \theta_{1}\left(\mathrm{r}^{\prime}\right)+2 v_{\mathrm{n} 1}^{0} \theta_{0}\left(\mathrm{r}^{\prime}\right)\right\}\left[\psi_{\mathrm{V} 1 \mathrm{~T} 1_{\mathrm{n} 1}}\left(\lambda-\mathrm{r}^{\prime}, \lambda-\mathrm{r}, \lambda\right)-\psi_{\mathrm{V} 1 \mathrm{~T} 1_{\mathrm{n} 1}}\left(\lambda, \lambda-\mathrm{r}, \lambda-\mathrm{r}^{\prime}\right)\right]
\end{aligned}
$$

Here $\mathrm{n}$ denotes the atomic species A and B. $\theta_{1}(\mathrm{r})$ is the step function which is unity when $\mathrm{r}=\mathrm{S}_{1}$ and zero otherwise. Similarly, $\theta_{0}(r)$ is the step function which is unity when $r=S_{0}$ and zero otherwise. To solve Eqn. 11 we use the simplest approximation, i.e., we express the atomic occupancy variables in terms of their average value and neglect the fluctuations from it. Furthermore we add and subtract terms with $r^{\prime}=r$ in the second term of Eqn. 11. After integrating with respect to time $t$ and summing over $\lambda$ we finally obtain:

$$
\begin{aligned}
& -\sum_{\lambda} \psi_{\mathrm{V} 1 \mathrm{~T} 1}(\lambda, \lambda-\mathrm{r} ; \mathrm{r}=0)=\left\{v_{\mathrm{T} 1}^{1} \theta_{1}(\mathrm{r})+2 v_{\mathrm{T} 1}^{0} \theta_{0}(\mathrm{r})\right\}\{\varphi(-\mathrm{r})-\varphi(\mathrm{r})\} \\
& +\sum_{\mathrm{n}} \mathrm{c}_{\mathrm{n} 1}\left\{v_{\mathrm{n} 1}^{1} \theta_{1}(\mathrm{r})+2 v_{\mathrm{n} 1}^{0} \theta_{0}(\mathrm{r})\right\} \varphi(\mathrm{r}) \\
& \quad+\sum_{\mathrm{n}} \mathrm{c}_{\mathrm{n} 1} \sum_{\mathrm{r}^{\prime}}\left\{v_{\mathrm{n} 1}^{1} \theta_{1}\left(\mathrm{r}^{\prime}\right)+2 v_{\mathrm{n} 1}^{0} \theta_{0}\left(\mathrm{r}^{\prime}\right)\right\}\left\{\varphi\left(\mathrm{r}-\mathrm{r}^{\prime}\right)-\varphi(\mathrm{r})\right\}
\end{aligned}
$$

Now, multiplying both sides by $\mathrm{e}^{\mathrm{ik.r}}$ and summing over $\mathbf{r}$, we soon get:

$$
\begin{aligned}
\tilde{\varphi}(\mathrm{k}:)=\frac{1}{v_{\mathrm{V} 1} \mathrm{D}(\mathrm{k})} & {\left[\sum_{\mathrm{r}} \mathrm{e}^{\mathrm{ik} . \mathrm{r}} \sum_{\lambda} \psi_{\mathrm{V} 1 \mathrm{~T} 1}(\lambda, \lambda-\mathrm{r} ; \mathrm{t}=0:)\right.} \\
& +\left(\frac{\mathrm{a}}{2 \pi}\right)^{3} \int \mathrm{d}^{3} \mathrm{k}^{\prime}\left\{v_{\mathrm{T} 1}^{1} \theta_{1}\left(\mathrm{k}+\mathrm{k}^{\prime}\right)+2 v_{\mathrm{T} 1}^{0} \theta_{0}\left(\mathrm{k}+\mathrm{k}^{\prime}\right)\right\} \tilde{\varphi}\left(\mathrm{k}^{\prime}:\right) \\
& \left.+\left(\frac{\mathrm{a}}{2 \pi}\right)^{3} \int \mathrm{d}^{3} \mathrm{k}^{\prime}\left\{\left(v_{\mathrm{V} 1}^{1}-v_{\mathrm{T} 1}^{1}\right) \theta_{1}\left(\mathrm{k}-\mathrm{k}^{\prime}\right)+2\left(v_{\mathrm{V} 1}^{0}-v_{\mathrm{T} 1}^{0}\right) \theta_{0}\left(\mathrm{k}-\mathrm{k}^{\prime}\right)\right\} \tilde{\varphi}\left(\mathrm{k}^{\prime}:\right)\right]
\end{aligned}
$$

where we have defined

and

$$
\mathrm{D}(\mathrm{k})=\left[\left\{\mathrm{Z}_{1}-\theta_{1}(\mathrm{k}\}+\frac{v_{\mathrm{V} 1}^{0}}{v_{\mathrm{v} 1}^{1}}\left\{\mathrm{Z}_{0}-2 \theta_{0}(\mathrm{k})\right\}\right]\right.
$$

$$
v_{\mathrm{V} 1}^{1(0)}=\mathrm{C}_{\mathrm{A} 1} v_{\mathrm{A} 1}^{1(0)}+\mathrm{C}_{\mathrm{B} 1} \mathrm{v}_{\mathrm{B} 1}^{1(0)}
$$


Here $v_{\mathrm{V} 1}^{1}$ and $v_{\mathrm{V} 1}^{0}$ are the average vacancy jump frequencies corresponding to the [110]-type and [100]-type sites respectively. Now, using Eqn. 13 in Eqn. 10a and following the procedure of Sandhu et al. [12], we get a linear equation in the U's as:

$$
\mathrm{a}_{11} \mathrm{U}_{0}+\mathrm{a}_{12} \mathrm{U}_{1}=\mathrm{V}^{(0)}
$$

where

$$
\begin{aligned}
\mathrm{V}^{(0)} & =8 \mathrm{i}\left(\frac{\mathrm{a}}{2 \pi}\right)^{3} \int \mathrm{d}^{3} \mathrm{k} \frac{\operatorname{sink}_{\mathrm{x}} \mathrm{a}}{\mathrm{D}(\mathrm{k})} \sum_{\mathrm{r}} \mathrm{e}^{\mathrm{ik} \cdot \mathrm{r}} \sum_{\lambda} \psi_{\mathrm{V} 1 \mathrm{~T} 1}(\lambda, \lambda-\mathrm{r} ; \mathrm{t}=0:) \\
\mathrm{a}_{11} & =\left\{v_{\mathrm{V} 1}^{1}-\frac{1}{2} \mathrm{~J}_{2}\left(v_{\mathrm{V} 1}^{0}-2 v_{\mathrm{T} 1}^{0}\right)\right\} \\
\mathrm{a}_{12} & =-\mathrm{J}_{1}\left\{v_{\mathrm{V} 1}^{1}-2 v_{\mathrm{T} 1}^{1}\right\}
\end{aligned}
$$

Since in the Manning [22,23] random alloy model, the evaluation of the static correlation functions is trivial, $\mathrm{V}^{(0)}$ can be evaluated easily for a given initial state. For example:

$$
\begin{aligned}
& \mathrm{V}^{(0)}\left(: \mathrm{S}_{1}^{0}\right)=-\mathrm{J}_{1} \mathrm{C}_{\mathrm{V} 1} \mathrm{C}_{\mathrm{T} 1} \\
& \mathrm{~V}^{(0)}\left(: \mathrm{S}_{0}^{0}\right)=-\mathrm{J}_{2} \mathrm{C}_{\mathrm{V} 1} \mathrm{C}_{\mathrm{T} 1}
\end{aligned}
$$

In the above, $\mathrm{J}_{1}$ and $\mathrm{J}_{2}$ are the integrals that can be obtained from

$$
J=8\left(\frac{a}{2 \pi}\right)^{3} \int d^{3} k \frac{\sin ^{2} k_{x} a}{D(k)} e(k)
$$

when the function $e(k)$ is replaced by $\cos \left(k_{y} a\right)$ and 1 respectively. In order to obtain $U_{0}$ and $U_{1}$ for a given initial configuration we need another equation in addition to Eqn. 16. Substitution of Eqn. 13 in Eqn. 10b, and following the above procedure, we get:

$$
\mathrm{a}_{21} \mathrm{U}_{0}+\mathrm{a}_{22} \mathrm{U}_{1}=\mathrm{V}_{1}^{(0)}
$$

where

$$
\begin{aligned}
& \mathrm{a}_{21}=-\frac{1}{2} \mathrm{~J}_{2}^{\prime}\left\{v_{\mathrm{V} 1}^{0}-2 v_{\mathrm{T} 1}^{0}\right\} \\
& \mathrm{a}_{22}=\left\{v_{\mathrm{V} 1}^{1}-\mathrm{J}_{1}^{\prime}\left(v_{\mathrm{V} 1}^{1}-2 v_{\mathrm{T} 1}^{1}\right)\right\}
\end{aligned}
$$

The function $\mathrm{V}_{1}^{(0)}$ can be obtained from $\mathrm{V}^{0}$ (Eqn. 17) if we replace $\sin \mathrm{k}_{\mathrm{x}} \mathrm{a}$ in the integrand by $\sin \mathrm{k}_{\mathrm{x}} \mathrm{a} \cos \mathrm{k}_{\mathrm{y}} \mathrm{a}$. Similarly, $\mathrm{J}_{1}^{\prime}$ and $\mathrm{J}_{2}^{\prime}$ can be obtained from the general integral $\mathrm{J}$ if we replace $\mathrm{e}(\mathrm{k})$ by $\cos ^{2} \mathrm{k}_{\mathrm{y}} \mathrm{a}$ and $\cos \mathrm{k}_{\mathrm{y}} \mathrm{a}$ respectively. Thus all the U's required in Eqn. 9 for the evaluation of the correlated part of the phenomenological coefficient can be obtained:

$$
\begin{aligned}
& U_{1}\left(: S_{1}^{0}\right)=-\frac{\left(a_{22} J_{1}-a_{12} J_{1}^{\prime}\right)}{\left(a_{11} a_{22}-a_{12} a_{21}\right)} C_{V 1} C_{T 1} ; U_{1}\left(: S_{0}^{0}\right)=\frac{\left(a_{22} J_{2}-a_{12} J_{2}^{\prime}\right)}{\left(a_{22} J_{1}-a_{12} J_{1}^{\prime}\right)} U_{1}\left(: S_{1}^{0}\right) \\
& U_{0}\left(: S_{1}^{0}\right)=-\frac{\left(a_{21} J_{1}-a_{11} J_{1}^{\prime}\right)}{\left(a_{22} J_{1}-a_{12} J_{1}^{\prime}\right)} U_{1}\left(: S_{1}^{0}\right) ; U_{0}\left(: S_{0}^{0}\right)=-\frac{\left(b_{1} J_{2}-a_{1} J_{2}^{\prime}\right)}{\left(a_{11} a_{22}-a_{12} a_{21}\right)} U_{1}\left(: S_{1}^{0}\right)
\end{aligned}
$$

The tracer correlation factor is given by:

$$
\mathrm{f}_{\mathrm{T} 1}=1+\frac{\mathrm{L}_{\mathrm{T} 1 \mathrm{~T} 1}^{(1)}}{\mathrm{L}_{\mathrm{T} 1 \mathrm{~T} 1}^{(0)}}
$$


Since the dynamics of the two sub-lattices is completely independent in the present model, the tracer correlation factor $\mathrm{f}_{\mathrm{T} 2}$ can be obtained from Eqn. 24 by inspection. The tracer correlation factor $\mathrm{f}_{\mathrm{T}}$ for the $\mathrm{B} 2$ intermetallic compound is then given by the harmonic mean of the two correlation factors as [17]:

$$
\frac{1}{\mathrm{f}_{\mathrm{T}}}=\frac{1}{2}\left(\frac{1}{\mathrm{f}_{\mathrm{T} 1}}+\frac{1}{\mathrm{f}_{\mathrm{T} 2}}\right)
$$

\section{Results and Discussion}

The Monte Carlo simulations for the four-frequency model have been carried out using standard procedures, see for example [15]. For a general review of Monte Carlo computer simulation of diffusion kinetics, see [24]. The equilibrium composition of A atoms was varied from 0.3 to 0.5 . The results for the tracer correlation factors $f_{A}$ and $f_{B}$ were obtained by varying the ratio of jump frequencies of regular and antistructural atoms, i.e., taking $\mathrm{W}_{\mathrm{A} 1}=\mathrm{W}_{\mathrm{B} 2}=1$ and $\mathrm{W}_{\mathrm{A} 2}=\mathrm{W}_{\mathrm{B} 1}=\alpha$ for different values of $\alpha$.

In order to compare the results of our expressions with those of the Monte Carlo simulation, we first consider the sublattice-1 which is the home sub-lattice for B atoms. As an approximation, we replace tracer frequencies by those of $\mathrm{A}$ and $\mathrm{B}$ atoms discussed in above. With this approximation, the frequencies $v_{\mathrm{T} 1}^{1}$ and $v_{\mathrm{T} 1}^{0}$ are equal and the $\mathrm{J}$ integrals become independent of frequencies. For example, when $\mathrm{T}=\mathrm{A}$, then $v_{\mathrm{T} 1}^{1}$ and $v_{\mathrm{T} 1}^{0}$ will be equal to the frequency of antistructural atom given by $2 \mathrm{JC}$; whereas for $\mathrm{T}=\mathrm{B}$, these will be equal to the frequency of the regular atom given by the 6JC mechanism, obtained through the procedure of Arita et al. [16]. The values of $\mathrm{J} 1$ and $\mathrm{J} 2$ turn out to be 0.0259 and 0.2416 , whereas those of $\mathrm{J}_{1}^{\prime}$ and $\mathrm{J}_{2}^{\prime}$ are 0.1252 and 0.0259 respectively.

The results of the tracer correlation factors for $A$ atoms are presented as a function of $C_{A}$ (near stoichiometry) for various values of $\alpha$ in Fig. 1a-d and for B atoms in Fig 2a-d. Our results (solid curves) are compared with Monte Carlo simulations (asterisks) and with those obtained by Sandhu et al. [12] (dashed curve). Our results are in reasonable agreement with the simulation results even when the ratio of the frequencies of the regular to antistructural atoms decreases to 0.1 . The agreement is better for $\mathrm{f}_{\mathrm{A}}$ compared with $\mathrm{f}_{\mathrm{B}}$. For a different comparison of the 6JC mechanism with the nearest neighbour vacancy jump approach, we have shown in Fig. 3 the dependence of $f_{A}$ on $\alpha$ for fixed values of $C_{A}$ covering the very wide range 0.3 to 0.5 . It is clear that the Monte Carlo values of correlation factors for the minority component (A) are largely independent of composition. However, this feature is not followed by the present results. The dependence of $f_{B}$ on $\alpha$ for fixed values of $C_{A}$ is shown in Fig. 4. In this case the Monte Carlo values for the majority component (B) show a strong dependence on composition. The present results show an even stronger dependence. Overall, it is interesting to note that the $6 \mathrm{JC}$ mechanism gives qualitatively similar results to that for the independent vacancy mechanism. It is noted that in Fig. 3 the terminal point for $\mathrm{f}_{\mathrm{A}}$ when $\alpha \rightarrow 0$ behaves in the same sort of manner as for the Monte Carlo simulation. The terminal point for $f_{B}$ is analogous to $f_{A}$ at the stoichiometric composition only. As $C_{A}$ decreases the terminal point for $\mathrm{f}_{\mathrm{B}}$ when $\alpha \rightarrow 0$ is no longer zero [15]. This is due to the fact that $\mathrm{B}$ is the majority component and B atoms will occupy a large portion of the two sub-lattices forming a network of B atoms [25]. The correlation factors of Sandhu et al. [12] become negative as the frequency ratio $\alpha$ in the range from 0.4 to 0.3 showing that the random alloy model is overestimating the tracer correlation effects when the formation of antistructural atoms is restricted. 
a
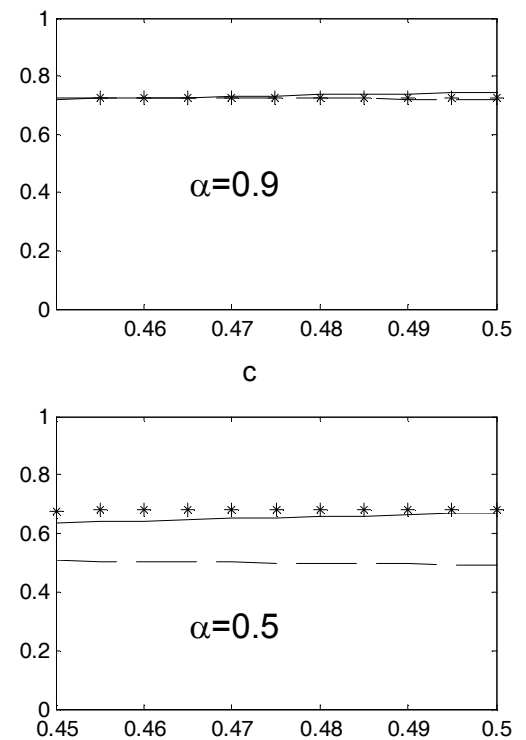

b
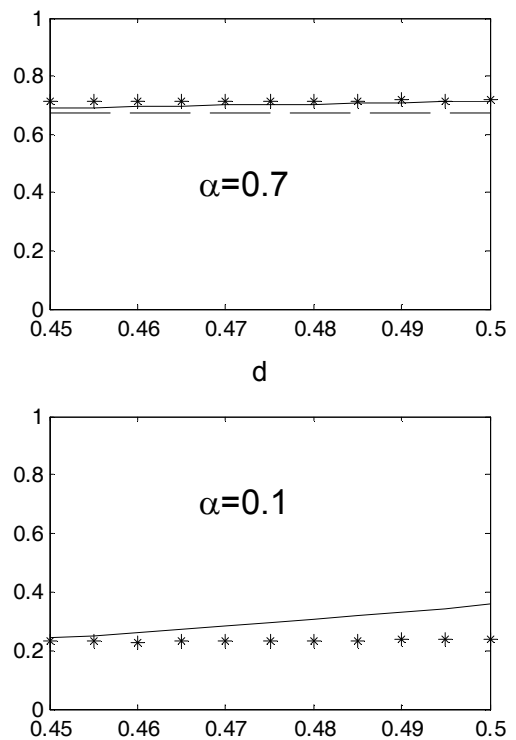

Fig. 1. Correlation factor $\mathrm{f}_{\mathrm{A}}$ as a function $\mathrm{C}_{\mathrm{A}}$ for (a) $\alpha=0.9$, (b) $\alpha=0.7$, (c ) $\alpha=0.5$ and (d) $\alpha=0.1$ Monte Carlo simulation -*, solid lines - results (present method), dashed lines - results of calculations by Sandhu et al.[12].
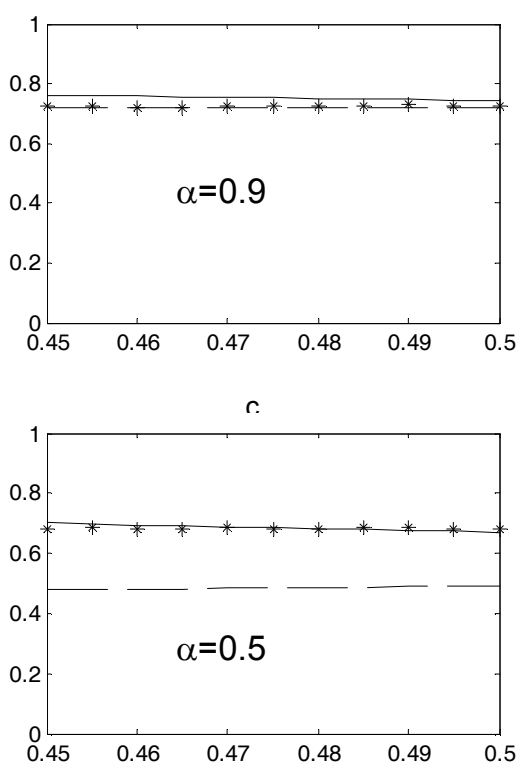

h

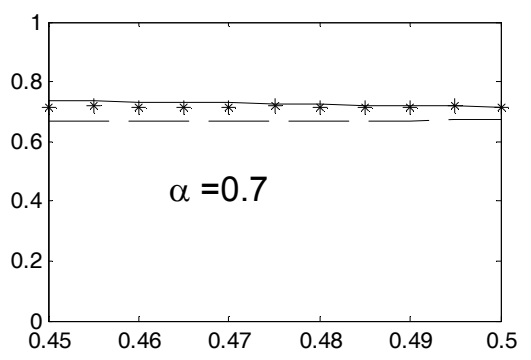

d

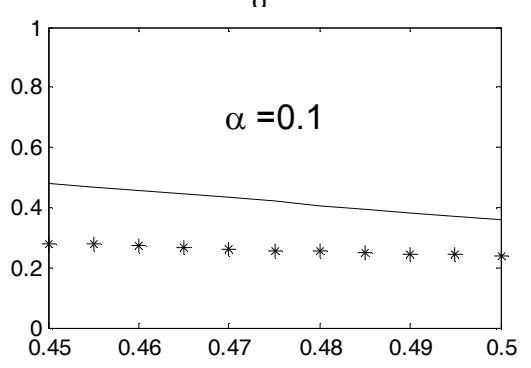

Fig. 2. Correlation factor $\mathrm{f}_{\mathrm{B}}$ as a function $\mathrm{C}_{\mathrm{A}}$ for (a) $\alpha=0.9$, (b) $\alpha=0.7$, (c ) $\alpha=0.5$ and (d) $\alpha=0.1$ Monte Carlo simulation -*, solid lines - results of calculations by present method, dashed lines - results of calculations by method of Sandhu et al. [12]. 


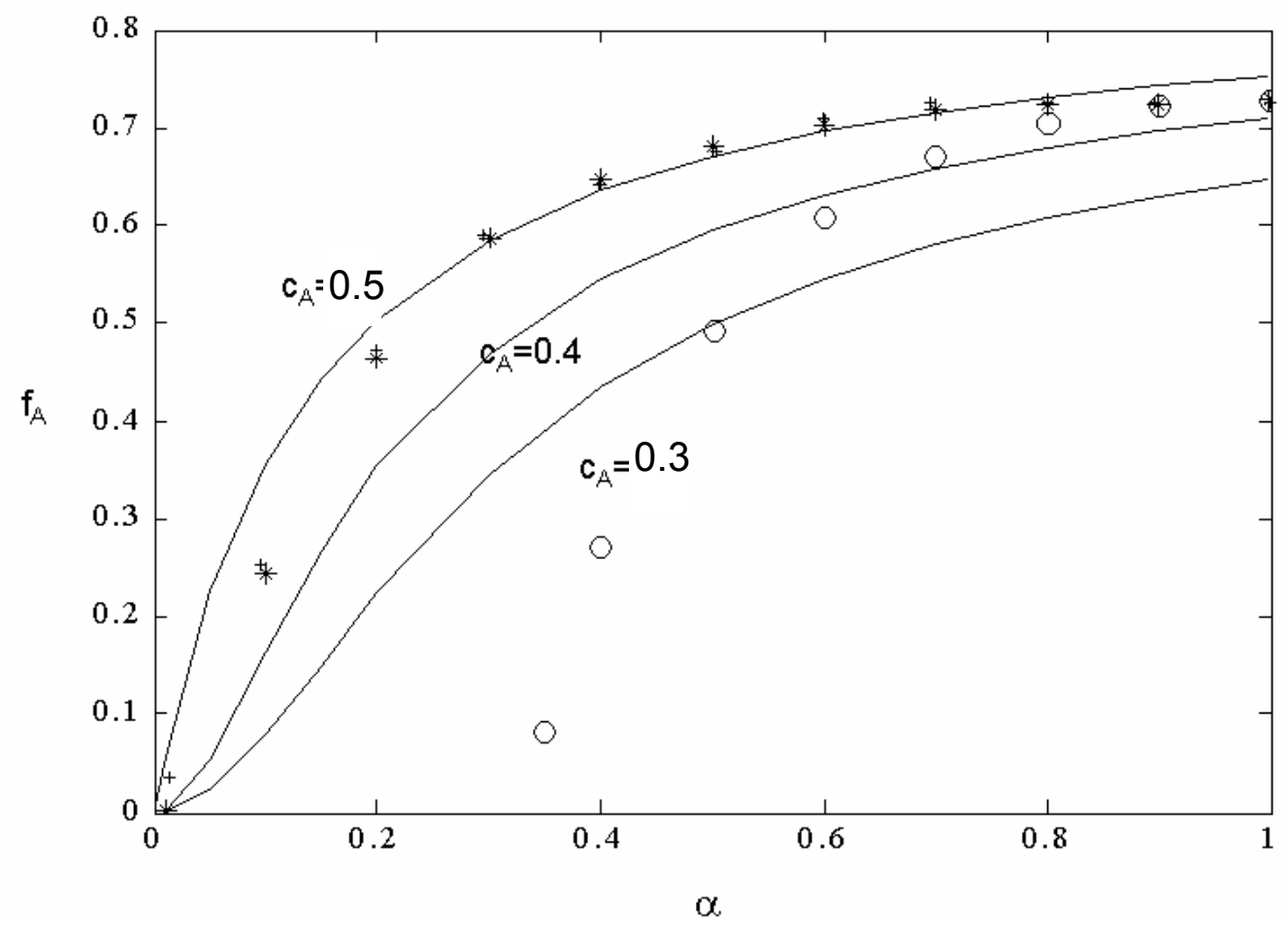

Figure 3. Correlation factor $\mathrm{f}_{\mathrm{A}}$ as a function of $\alpha$ for the $\mathrm{B} 2$ intermetallic compound. Solid line: present calculations for $\mathrm{C}_{\mathrm{A}}=0.3,0.4,0.5$. Symbols: Monte Carlo simulation, ${ }^{*}$ - for $\mathrm{C}_{\mathrm{A}}=0.5 ; \mathrm{x}-\mathrm{C}_{\mathrm{A}}=0.4 ;+-\mathrm{C}_{\mathrm{A}}=0.3 ; \mathrm{o}$ - results calculated by method described in Sandhu et al. [12] for $\mathrm{C}_{\mathrm{A}}=0.5$.

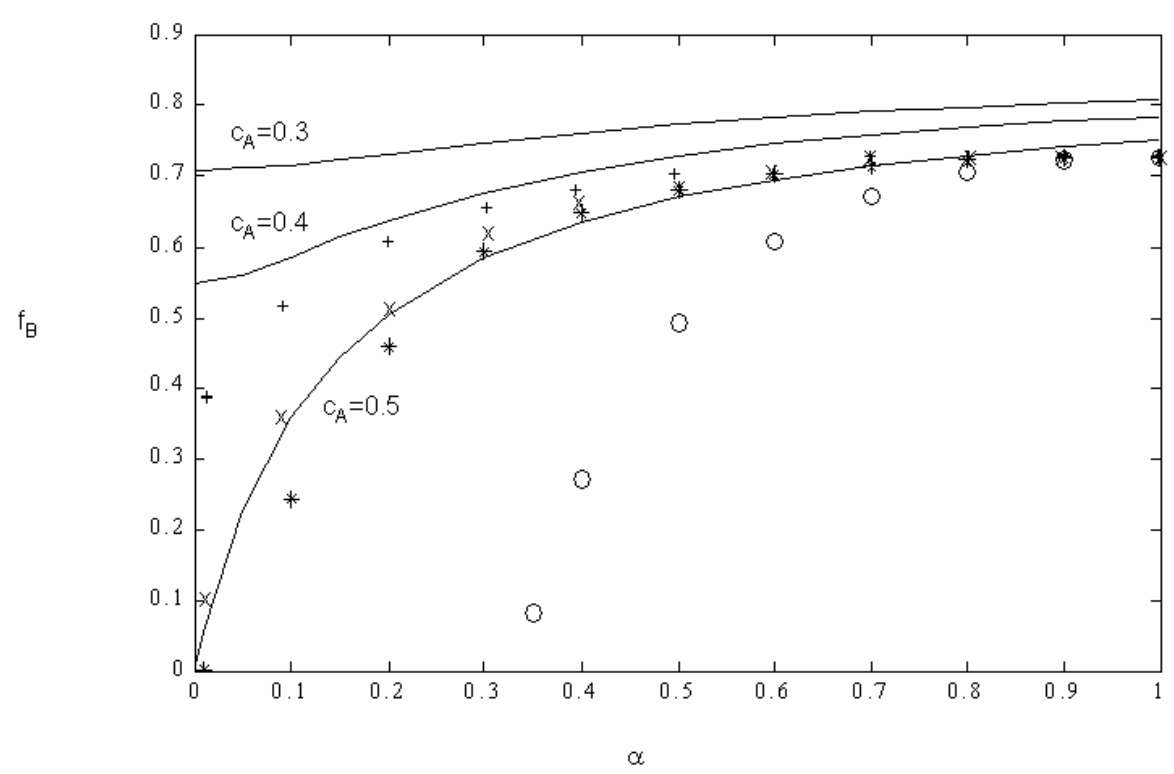

Figure 4. Tracer correlation factor $\mathrm{f}_{\mathrm{B}}$ as a function of $\alpha$ for the $\mathrm{B} 2$ intermetallic compound. Solid line: present calculations for $\mathrm{C}_{\mathrm{A}}=0.3,0.4,0.5$. Symbols: Monte Carlo simulation, * - for $\mathrm{C}_{\mathrm{A}}=0.5 ; \mathrm{x}-\mathrm{C}_{\mathrm{A}}=0.4 ;+-\mathrm{C}_{\mathrm{A}}=0.3$; o results calculated by method described in Sandhu et al. [12] for $\mathrm{C}_{\mathrm{A}}=0.5$. 


\section{Conclusions}

Tracer diffusion in non-stoichiometric B2 intermetallic compounds having antistructural disorder was investigated using the $6 \mathrm{JC}$ as a fundamental diffusion unit. For nonstoichiometric compositions, the antistructural atoms are assumed to be isolated and located at one of the six [110]-type and [100]-type sites (as only these sites are involved in the 6JC or $2 \mathrm{JC}$ ). The jump frequencies for the $6 \mathrm{JC}$ involving a perfectly ordered configuration were calculated in terms of a four-frequency-model, using the mean-first-passage concept of Arita et al. [16]. The jump frequency of an antistructural atom at [110] or [100]-type sites was taken to be the harmonic mean of frequencies of two successive nearest-neighbour jumps of the same kind of atoms. The expressions for the tracer diffusion coefficients were derived for both atomic components at deviations from stoichiometry, assuming that the $6 \mathrm{JC}$ mechanism is valid. The results were compared with Monte Carlo simulations based on single vacancy jumps and found to be in fair agreement for compositions close to stoichiometry.

\section{Acknowledgments}

One of us (KLG) is grateful to the University Grants Commission, New Delhi for the award of a 'Teacher Fellowship' and wishes to thank the Principal, Dyal Singh College, Karnal and Commissioner, Higher Education, Haryana for sanctioning the study leave. We also wish to thank the Australian Research Council for its support of this research.

\section{REFERENCES}

[1] J.H. Westbrook and R.L. Fleischer, Intermetallic Compounds: Principles and Practice (Wiley, New York, 1995).

[2] H.B. Huntingdon, N.C. Miller and V. Nersis, Acta Metall., 9 (1961), p.749.

[3] G.E. Murch and I.V. Belova, Mater. Res. Soc. Symp. Proc., 527, (1998) p.159.

[4] I.V. Belova and G.E. Murch, Defect and Diffusion Forum, 237-240 (2005), p.291.

[5] I.V. Belova, M.E. Ivory and G.E. Murch, Phil. Mag. A, 72 (1995) p.871.

[6] M. Athenes, P. Bellon and G. Martin, Phil. Mag. A, 76 (1997) p.565.

[7] H.A. Domian and H.I. Aaronson, Diffusion in Body Centred Cubic Metals, edited by

J.A. Wheeler and F.R. Winslow (ASM, Metals Park OH 1965), p. 209.

[8] I.V. Belova and G.E. Murch, Phil. Mag. A, 82 (2002), p.269.

[9] I.V. Belova and G.E. Murch, Phil. Mag. A, 82 (2002), p. 285.

[10] I.V. Belova and G.E. Murch, J. Phys. Condens. Matter, 14 (2002), p. 9563.

[11] A.R. Allnatt and A.B. Lidiard, Atomic Transport in Solids (Cambridge University Press, 1993).

[12] I.S. Sandhu, D.K. Chaturvedi, I.V. Belova and G.E. Murch, Defect and Diffusion Forum, accepted and in press. 
[13] H. Sato, Physical Chemistry: An Advanced Treatise, Vol. 10, edited by H. Eyring, D.Henderson

and W. Jost (Academic Press, NY, 1971), p. 579.

[14] H. Sato, T. Ishikawa and R. Kikuchi, J. Phys. Chem. Solids, 46 (1985), p.1361.

[15] I.V. Belova and G.E. Murch, Phil. Mag. A, 73 (1996), p.117.

[16] M. Arita, M. Koiwa and S. Ishioka, Acta Metall., 37 (1989), p.1363.

[17] H. Bakker, Phil. Mag. A, 40 (1979), p.525.

[18] D.K. Chaturvedi and A.R. Allnatt, Phil. Mag. A, 69 (1994), p.82.

[19] D.K. Chaturvedi and A.R. Allnatt, Phil. Mag. A, 54 (1994), p.1498.

[20] S. Sharma, R. Singh and D.K. Chaturvedi, Phil. Mag. A, 79 (1999), p.2083.

[21] S. Sharma, D.K. Chaturvedi, I.V. Belova and G.E. Murch, Phil. Mag. A, 80 (2000), p.65.

[22] J.R. Manning, Diffusion Kinetics for Atoms in Crystals (Van Nostrand, Princeton NJ 1968).

[23] J.R. Manning, Phys. Rev. B, 4 (1971), p.1111.

[24] G.E.Murch, Diffusion in Crystalline Solids, edited by G.E. Murch and A.S. Nowick (Academic Press, Orlando FL 1984), p.379.

[25] I.V. Belova and G.E. Murch, Intermetallics, 6 (1998), p. 115. 
Defects and Diffusion in Metals - An Annual Retrospective VIII doi:10.4028/3-908451-20-5

Tracer Diffusion by Six-Jump-Cycles in Nonstoichiometric B2 Intermetallic Compounds

doi:10.4028/3-908451-20-5.9 